\title{
Low-density InAs QDs with subcritical coverage obtained by conversion of In nanocrystals
}

\author{
Adam Urbańczyk , Richard Nötzel
}

\section{A R T I C L E I N F O}

Keywords:

A1. Nanostructures

A3. Molecular beam epitaxy

B2. Semiconducting III-V materials

\begin{abstract}
A B S T R A C T
We report growth of InAs/GaAs quantum dots (QDs) by molecular beam epitaxy with low density of $2 \mu \mathrm{m}^{-2}$ by conversion of In nanocrystals deposited at low temperatures. The total amount of InAs used is about one monolayer, which is less than the critical thickness for conventional Stranski-Krastanov QDs. We also demonstrate the importance of the starting surface reconstruction for obtaining uniform QDs. The QD emission wavelength is easily tunable upon post-growth annealing with no wetting layer signal visible for short anneals. Microphotoluminescence measurements reveal well separated and sharp emission lines of individual QDs.
\end{abstract}

\section{Introduction}

InAs/GaAs quantum dots (QDs) are prime candidates for solidstate realization of functionalities such as qubits [1], quantum gates [2], single photon sources [3,4], and cavity quantum electrodynamics systems [5]. Such applications require reproducible and well controllable means of obtaining low-density QDs, as a single QD is the basic building block of the above mentioned devices. Low-density InAs/GaAs QDs can be obtained by molecular beam epitaxy (MBE) using the Stranski-Krastanov (SK) growth mode and small InAs coverage above the critical thickness [6] or extremely small growth rates [7]. An alternative, yet so far widely unexplored route to achieve this is to use droplet epitaxy [8-10], where one first deposits metallic liquid In droplets or, in our case epitaxial In nanocrystals and afterwards recrystalizes them under As flux. This offers a very robust and reproducible way to grow low-density QDs without relying on extremely low flux or precise coverage control.

Here we report the growth of low-density InAs/GaAs QDs by conversion of In nanocrystals. We routinely obtain densities down to $2 \mu \mathrm{m}^{-2}$. We show the importance of the starting surface reconstruction for the growth of In nanocrystals and their subsequent conversion to InAs QDs. For a starting $(2 \times 4)$ or less As rich surface reconstruction the QDs are uniform and the total InAs coverage necessary to obtain them is one monolayer (ML), significantly less than the critical thickness for SK QDs. The QDs after capping emit around $1 \mu \mathrm{m}$, tunable upon post-growth annealing. Up to high excitation power there is no wetting layer signal visible for short anneals. Microphotoluminescence (microPL) measurements reveal sharp and well separated emission lines from individual QDs.

\section{Experimental details}

All samples were grown by solid-source MBE on singular (100) oriented, undoped GaAs substrates. After oxide removal under As 4 flux at $580^{\circ} \mathrm{C}$ a $200 \mathrm{~nm} \mathrm{GaAs}$ buffer layer was grown. The substrates were then cooled to $420^{\circ} \mathrm{C}, 350^{\circ} \mathrm{C}$, or $250^{\circ} \mathrm{C}$ where the As flux was switched off. For the highest temperature a clear $(2 \times 4)$ surface reconstruction was observed by reflection high energy electron diffraction (RHEED), while for the lower temperatures the surface reconstruction was $c(4 \times 4)$. The $(2 \times 4)$ reconstruction was most likely the gamma phase (see inset in Fig. 1). The substrates were then cooled to $100^{\circ} \mathrm{C}$ with the base pressure in the growth chamber below $2 \times 10^{-8} \mathrm{mbar}_{\text {. The }} \mathrm{As}_{4}$ background pressure was low enough that the respective surface reconstructions were stable for at least $30 \mathrm{~min}$, also in the $(2 \times 4)$ case. Indium was deposited at a growth rate of $0.01 \mathrm{ML} / \mathrm{s}$ to form In nanocrystals with well defined epitaxial relationship, evidenced by the observation of a spotty RHEED transmission 
a
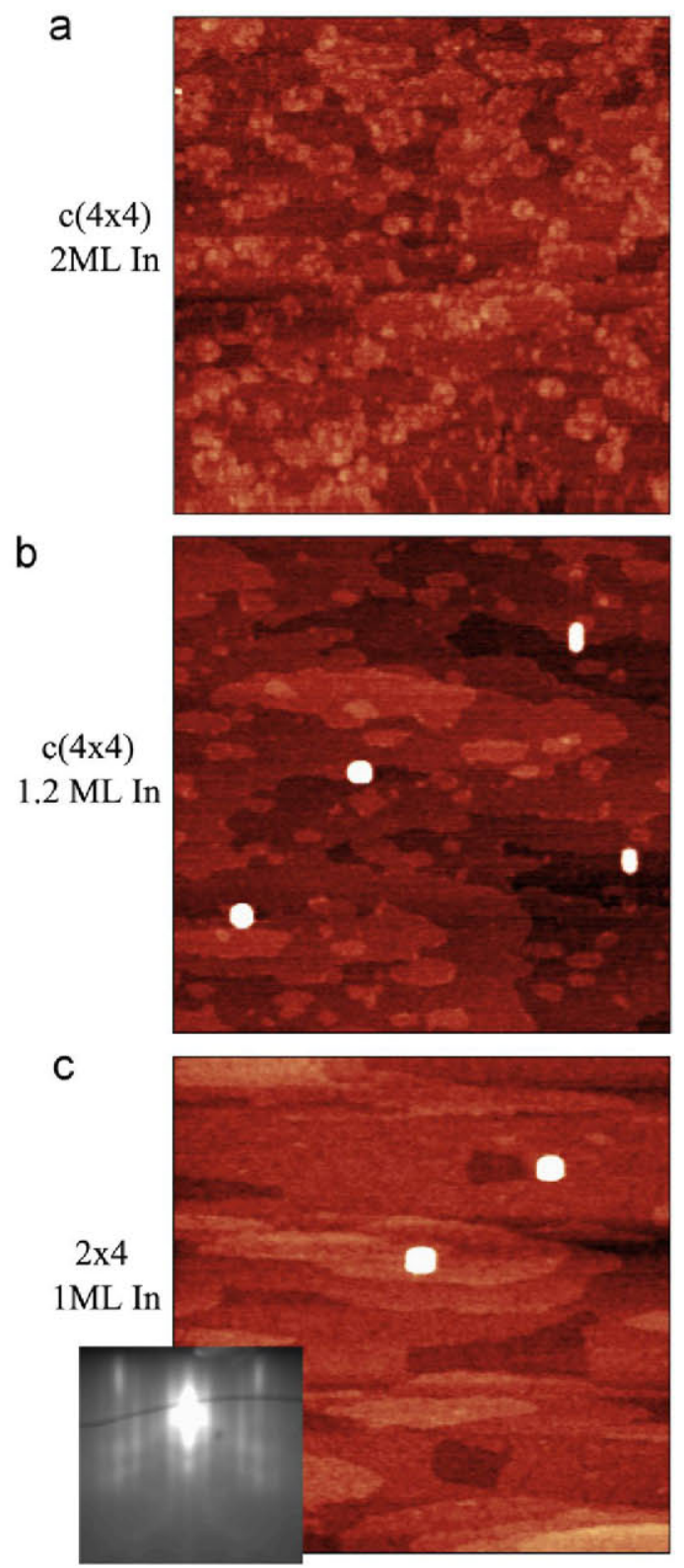

Fig. 1. Influence of the initial surface reconstruction and stoichiometry on the morphology of In nanocrystals observed by AFM. The height contrast is $3 \mathrm{~nm}$ and all scan fields are $1 \mu \mathrm{m} \times 1 \mu \mathrm{m}$. (a) As supply switched off at $250^{\circ} \mathrm{C}$, starting surface reconstruction $c(4 \times 4)$, no RHEED transition observed for In coverage up to $2 \mathrm{ML}$. (b) As supply switched off at $350^{\circ} \mathrm{C}$, starting surface reconstruction $\mathrm{c}(4 \times 4)$, RHEED transition visible for In coverage of $1.2 \mathrm{ML}$. (c) As supply switched off at $420^{\circ} \mathrm{C}$, starting surface reconstruction $(2 \times 4)$, RHEED transition visible for In coverage of $1 \mathrm{ML}$. Inset in (c) shows a RHEED pattern in [0-11] direction captured directly before the deposition of In.

diffraction pattern (reported previously in [10]). The nanocrystals were transformed to InAs QDs by annealing under $\mathrm{As}_{4}$ flux, initially at $100{ }^{\circ} \mathrm{C}$ and then at $420^{\circ} \mathrm{C}$ for $10 \mathrm{~min}$. Samples for optical characterization were additionally heated up to $480^{\circ} \mathrm{C}$, annealed for 1 or $10 \mathrm{~min}$, capped with $20 \mathrm{~nm}$ GaAs, then heated to $580^{\circ} \mathrm{C}$, and finally capped with $100 \mathrm{~nm}$ GaAs. The morphology of the samples was characterized by tapping-mode atomic force microscopy (AFM) under ambient conditions. The density of the QDs was determined based on $8 \times 8 \mu \mathrm{m}^{2}$ images to evaluate a statistically significant QD number. Photoluminescence (PL) measurements were performed with the samples placed in a continuous-flow He cryostat at $10 \mathrm{~K}$. A long working distance objective (0.5 numerical aperture) was used to excite the samples and collect the emitted light. A $630 \mathrm{~nm}$ semiconductor laser was used as an excitation source. The PL was dispersed by a monochromator and detected by a liquid-nitrogen cooled InGaAs photodiode array.

\section{Results and discussion}

The GaAs surface reconstruction, which is directly linked with the surface stoichiometry [11], depending on temperature and As flux, has profound impact on the growth of In nanocrystals, as presented in Fig. 1. For the most As-rich starting surface, with the As flux stopped at $250^{\circ} \mathrm{C}$, no In nanocrystals form for an In coverage up to $2 \mathrm{ML}$ and the resulting surface is very rough (Fig. 1(a)). All In reacts with excess As to form an irregular InAs layer. For the intermediate case when the As supply is switched off at $350{ }^{\circ} \mathrm{C}$ In nanocrystals form for $1.2 \mathrm{ML}$ In deposition (Fig. 1(b)). Interestingly, they have a clearly bimodal size distribution. $80 \%$ have a square shape with average height of $15 \mathrm{~nm}$ and base size of $40 \mathrm{~nm}$. The remaining $20 \%$ have an elongated shape with long-axis parallel to [011], average height of $5 \mathrm{~nm}$ and average length and width of $90 \mathrm{~nm}$ and $35 \mathrm{~nm}$, respectively. Careful analysis of Fig. 1(b) reveals, that the elongated islands are connected to traces parallel to [011], what is visible in more detail in Fig. 2(b). This observation is analogous to findings of Tersoff et. al. [12]. Most likely the driving force for elongation is reaction of excess As on the surface with metallic In leaving behind those traces. In case of the less As-rich $(2 \times 4)$ starting surface reconstruction studied here, prepared by switching off the As supply at $420^{\circ} \mathrm{C}$, the RHEED transition indicating

a

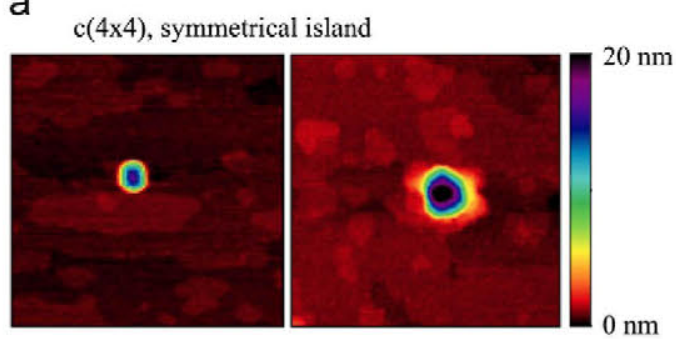

b c(4x4), elongated island

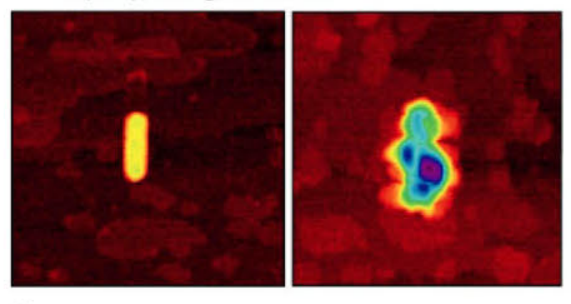

C $2 \times 4$
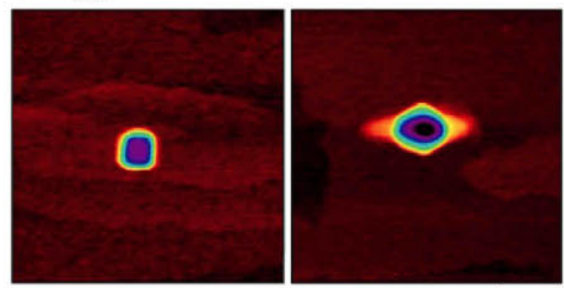

$400 \mathrm{~nm}$

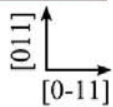

Fig. 2. AFM images of In nanocrystals before (left images) and after annealing for $10 \mathrm{~min}$ at $420^{\circ} \mathrm{C}$ in As flux (right images). (a) $\mathrm{c}(4 \times 4)$ starting surface reconstruction, symmetric In nanocrystal, (b) $c(4 \times 4)$ starting surface reconstruction, elongated In nanocrystal, $(c)(2 \times 4)$ starting surface reconstruction. 


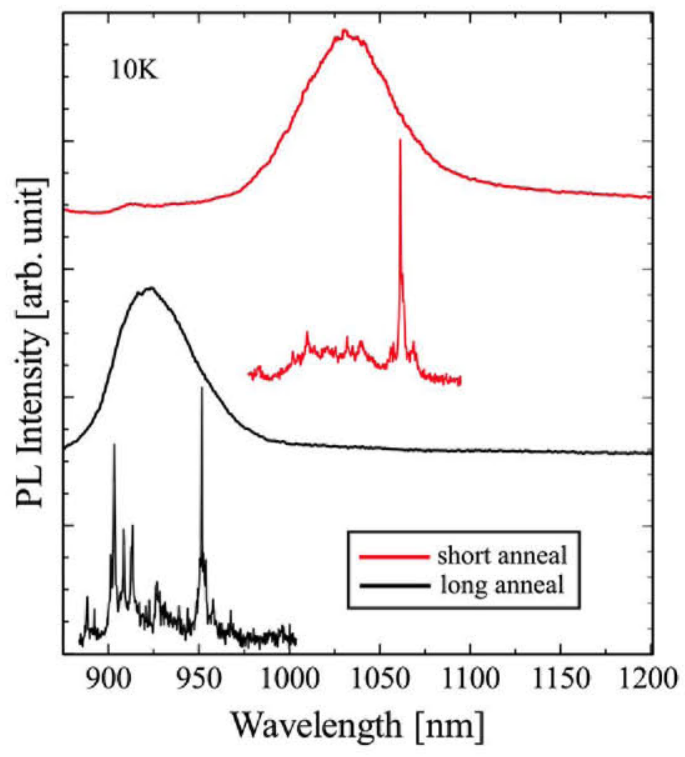

Fig. 3. Low-temperature PL and micro-PL spectra of capped QDs annealed for 1 and $10 \mathrm{~min}$ at $480^{\circ} \mathrm{C}$. Curves are normalized and vertically offset for clarity.

In nanocrystal formation is observed for In coverages as low as $1 \mathrm{ML}$. Moreover, the observed surface roughness is lowest of all three cases. It has to be noted that the initial surface reconstruction does not influence the observed spotty RHEED pattern of the In nanocrystals, which means that it does not have any influence on the In/GaAs crystallinity and epitaxial relationship. The In nanocrystals in case of the $(2 \times 4)$ reconstruction (Fig. 1(c)) have a uniform square shape with average base size of $55 \mathrm{~nm}$ and mean height of $17 \mathrm{~nm}$. Clearly there is a trend of decreasing critical coverage for In nanocrystal formation with decreasing the amount of As on the initial GaAs surface. This is understandable, as excess As is first converted to InAs by impinging In atoms before metallic In nanocrystals can form. The additional InAs does not form a smooth layer, but contributes to the increased roughness of the surface.

Fig. $2(\mathrm{a}-\mathrm{c})$ shows the conversion of individual In nanocrystals on the $\mathrm{c}(4 \times 4)$ and $(2 \times 4)$ reconstructed GaAs surfaces (left images) to InAs annealed at $420{ }^{\circ} \mathrm{C}$ (right images). There is oneto-one In nanocrystal to InAs island correspondence, because the initial and final densities match. In accordance with previous reports [10], the InAs islands flatten and develop a local wetting layer elongated along $[0-11]$. For the symmetric In nanocrystals on the $c(4 \times 4)$ reconstructed surface (Fig. 2(a)) and in particular for the elongated In nanocrystals (Fig. 2(b)) this results in a very distorted shape of the InAs islands and local wetting layer. Prolonged annealing and/or annealing at higher temperature leads to extension of the local wetting layer, further flattening of the QDs and finally complete collapse with formation of a quasi two-dimensional layer (not shown here). This is understood by the fact, that the total InAs coverage is lower than the critical thickness for QD formation in the SK growth mode, hence, the InAs islands are not stable. This, however, provides a very reproducible and robust means of controlling QD size and PL emission wavelength upon post-growth annealing, which we show now for the symmetric QDs (Fig. 2(c)) obtained on the $(2 \times 4)$ reconstructed $\mathrm{GaAs}$ surface.

PL measurements performed at $10 \mathrm{~K}$ of the capped QD samples annealed for 1 and $10 \mathrm{~min}$ at $480^{\circ} \mathrm{C}$ reveal clear emission peaks centered at 1030 and $925 \mathrm{~nm}$, respectively, shown in Fig. 3. No wetting layer signal is observed at shorter wavelength for the $1030 \mathrm{~nm}$ sample, even at high excitation power density of $5 \mathrm{~kW} / \mathrm{cm}^{2}$, indicating the absence of an optically active wetting layer. This is important in view of more ideal zero-dimensional nanostructures with minimized interaction with their surrounding $[13,14]$. For long anneals a PL signal at shorter wavelength attributed to the local wetting layer becomes visible, what is consistent with the AFM observation of increasing local wettinglayer with increasing annealing time. Micro-PL measurements, presented in Fig. 3, show sharp emission lines from individual QDs with linewidths down to $30 \mu \mathrm{eV}$, which is the resolution limit of the used setup. This evidences that our approach of converting In nanocrystals to InAs QDs, deposited on As-poor GaAs surfaces, reproducibly provides high quality QDs of low density required for applications relying on single QDs.

\section{Conclusions}

In conclusion, we demonstrated growth of QDs with low density of $2 \mu \mathrm{m}^{-2}$ by conversion of In nanocrystals into InAs after annealing under As flux. The initial morphology of the In nanocrystals depended strongly on the starting surface reconstruction and it was found that the most uniform structures were obtained for the $(2 \times 4)$ surface reconstruction. In this case the total InAs coverage for QD formation amounted to $1 \mathrm{ML}$, which is significantly less than the InAs critical thickness for QDs grown in the SK mode. Capped QDs emitted around $1 \mu \mathrm{m}$ at low temperature, widely tunable upon post-growth annealing. No wetting layer emission was observed for samples with short annealing time. Micro-PL measurements revealed sharp and well isolated emission lines from individual QDs.

\section{References}

[1] D. Press, T.D. Ladd, B. Zhang, Y. Yamamoto, Nature 456 (2008) 218.

[2] X. Li, Y. Wu, D. Steel, D. Gammon, T.H. Stievater, D.S. Katzer, D. Park, C. Piermarocchi, L.J. Sham, Science 301 (2003) 809.

[3] R.M. Stevenson, R.J. Young, P. Atkinson, K. Cooper, D.A. Ritchie, A.J. Shields, Nature 439 (2006) 179.

[4] S. Laurent, S. Varoutsis, L.L. Gratiet, A. Lemaitre, I. Sagnes, F. Raineri, A. Levenson, I. Robert-Philip, I. Abram, Applied Physics Letters 87 (2005) 163107.

[5] A. Badolato, K. Hennessy, M. Atature, J. Dreiser, E. Hu, P.M. Petroff, A. Imamoglu, Science 308 (2005) 1158.

[6] N.P. Kobayashi, T.R. Ramachandran, P. Chen, A. Madhukar, Applied Physics Letters 68 (1996) 3299.

[7] B. Alloing, C. Zinoni, L.H. Li, A. Fiore, G. Patriarche, Journal of Applied Physics 101 (2007) 024918.

[8] T. Chikyow, N. Koguchi, Japanese Journal of Applied Physics 29 (1990) L2093.

[9] J.S. Kim, N. Koguchi, Applied Physics Letters 85 (2004) 5893.

[10] A. Urbanczyk, G.J. Hamhuis, R. Notzel, Journal of Applied Physics 107 (2010) 014312.

[11] C. Deparis, J. Massies, Journal of Crystal Growth 108 (1991) 157

[12] J. Tersoff, D.E. Jesson, W.X. Tang, Science 324 (2009) 236.

[13] A. Vasanelli, R. Ferreira, G. Bastard, Physical Review Letters 89 (2002) 216804.

[14] C. Kammerer, C. Voisin, G. Cassabois, C. Delalande, P. Roussignol, F. Klopf, P. Reithmaier, A. Forchel, J.M. Gerard, Physical Review B 66 (2002) 041306. 